\title{
Neuroengineering lessons from a philosophy of biology
}

\author{
Denis Larrivee* \\ International Association Catholic Bioethicists, Canada
}

Driven by a barrage of neuroscientific and neuroengineering findings, from functional features such as metastability indices and frontoparietal activation [1,2], therapeutic strategies like myelin regrowth and cell cycle activators [3], or mobilized assistive technologies [4], perceived prospects for neurorehabilitative outcome have entered a phase that, while not ebullient, nonetheless shimmers with anticipation. By comparison, prospects for retrieving normal mobility in the 1970s and 1980s for patients with severe lumbar trauma, let alone cervical transection or higher cognitive impairment, were minimal to non-existent. Basic research initiatives undertaken then by private funding organizations like the Paralyzed Veterans of America, for example, focused on endogenous cellular mechanisms affecting process outgrowth and synaptic targeting in spinal or peripheral nerves [5]. Advances in basic research since, on most aspects of nervous system function, from single neuron to large scale multi network modules, have greatly improved this understanding. Significantly, this basic research understanding has collectively synergized with microengineering developments to expand the prospective range for neurotechnology intervention in the form of medical implant devices [6].

This latter is good news for patients, especially the swelling ranks of those past 60 years of age that World Health Organization demographics portend [7]. Improvements in medical care have today significantly extended life expectancies, increasing population percentages of this age group in a majority of nations around the globe that are projected to reach $25 \%$ or better in the most technologically advanced nations. Dominating the health prognostications of this population sector, however, is the considerably increased risk of cognitive impairment; hence, also the increased likelihood of the long term medical health care burden associated with it. WHO projections, for example, indicate that the progressively increasing percentage of those within this group who have suffered cerebral ischemia has been amplified by the substantially reduced mortality to incidence ratio [8], making long term therapeutic assistance for substantially greater numbers of patients nearly inevitable.

Early approaches to neural implant devices appealed to the relatively simpler anatomical configuration and functioning of peripheral sensory and motor nerves, and the basic to and from communication mediated between brain and body of the spinal cord. This simplicity essentially limited the spectrum of technical obstacles that faced researchers then. Therapeutic objectives of the devices were thus confined to the replacement of lost neural function through a mimicking of neural signals that would ordinarily be transmitted in the damaged peripheral neural cells to their respective effector targets. Jensen, Andersen, and Akay's text title from the $2^{\text {nd }}$ International Neurorehabilitative conference summarizes this basic thematic approach to design with "Replace, Repair, Restore, Relieve" [9]. Interfacial design for implants has thus been premised on an understanding of nerve function that is linear with respect to the production of nerve signals and delivered directly to an end target. In the absence of signals the presupposition has been the absence of meaningful activity, a functional presupposition that would require a direct replacement of Hodgkin Huxley spiking activity.

Adopting this interface design strategy, neural implant devices have been successfully developed for a wide variety of neural impairments affecting the peripheral nervous system, including, for example, cochlear and retinal implants, pacemakers, vagal stimulators, muscle stimulators, and the like. Indeed, the principal technical difficulties for device implementation have been related to signal distortion at high frequency due to the high impedance and capacitance of the microelectrodes used in recording [10]. Refinements in electrode construction and improvements in circuit design, moreover, have increasingly lessened the significance of even this signal sensing loss to implant function.

Building on such earlier designs, implant devices for the brain have acquired a design legacy that, while acknowledging the brain's greater anatomical complexity, remains, nonetheless, a direct extrapolation of that premised in the interfacial designs of peripheral implants. Illustrative examples include the use of the local field potential, a parameter measuring the electrical contribution of all cellular elements within a microvolume of tissue, which is exploited for the purpose of isolating spiking rates from individual neurons [11].

Neglected in presuppositions underpinning these strategies, however, is a synthetic philosophy of science perspective that attempts to reconcile information flow in the brain with the brain's global role in guiding organismal behavior. Humans, notably, like all organisms, are autonomous, a capacity mediated in large measure by the brain, which is to say that the brain has been evolutionarily crafted to enable autonomy, and not vice versa. Moreover, humans and other organisms are endowed with goal directedness, meaning that the whole organism, within the confines of its topological perimeters, is subordinate to the brain's (and peripheral nervous system's) guidance. Due to these supraphysical constraints a number of consequences ensue, that differentiate the brain's organization and operation from a strictly feed forward, off on off working arrangement used in peripheral nerves for direct communication with an effector organ..

For example, the brain assists in maintaining bodily homeostasis, and so is, necessarily, persistently active [12]. There is, thus, a constant stream of sensorial input as well as interareal communication that generates a prevalent, indeed persistent, condition of stochastic noise.

Correspondence to: Denis Larrivee, International Association Catholic Bioethicists, Toronto, Canada, E- mail: sallar1@aol.com

Received: October 01, 2017; Accepted: October 17, 2017; Published: September 21,2017 
Transmission of signals through nerve networks, accordingly, always includes a mixture of signal and noise. For a feedforward architecture this means that functions of state, such as that resulting from signal output, are continually rising, albeit in an irregular and stochastic manner[13]. Accordingly, the brain's anatomy must be configured to overcome this obstacle, an organization that is achieved through recurrent connectivity - indeed, nearly $95 \%$ of brain neurons possess recurrent connections. Using this configuration nerve signals are cycled through preferred, low resistance pathways that help to create stable and physiologically reliable response architectures that can overcome the noisy background in which they are immersed.

It also means that output is no longer a direct function of input, that is, it is no longer directly related to spiking activity occurring in brain neurons, as can be seen in Usher-McClelland classification networks [14]. In this simple connectivity arrangement single neuron units are self-excitatory while inhibitory to all other feed forward neurons. Steady inputs here do not yield a constantly rising output that is linear with respect to input but rather one that asymptotically reaches a steady state; that is, over a limited amount of time the response dynamics becomes independent of input. A system of constraints is thus established that dynamically stabilizes the signal trajectory, termed an attractor motif that becomes instead the physiologically relevant parameter for the execution of behavior, a circumstance that bears significance for implant design.

Accessing these elements is thus the critical dimension in eliciting their physiological use. Because attractors are fundamentally structured to attain to stable functions of state, access occurs at their entry, which thus becomes the mode of engaging these dynamic 'motifs'. Entry constitutes a critical, decision event, that is, an energetically favorable comparative assessment that enables access to the attractor [15]. Trajectories through the attractor may subsequently encounter local instabilities, that is, further decision points where they may leave the attractor, with the potential of entering still another dynamical motif. Leveraging instabilities, accordingly, offers a modus for exercising control over implementation of the attractor and a way of generating combinatorial variation. Guiding the trajectory through the dynamic space thus becomes critical to the implementation of the interfacial device.

This combinatorial and hierarchical scaling of dynamical elements offers an explanation for yet another fundamental feature of living systems. That is, how they behave as goal directed entities. Integrated goal seeking, notably, constitutes the ultimate objective of living systems needed for autonomy, and dynamic elements offer the means to achieving this goal. Global systems help to ensure global identity and to achieve integrated and coordinated activity. In primitive organisms like $\mathrm{C}$ elegans, for example, there is an intimate association between global dynamics and motor effectors [16]. Similarly, in humans it is necessary to construct a 3 dimensional self-image as a coherent percept to generate both motor planning trajectories and motor activity [17].

These biological imperatives make the task of designing a brain implant interface for neurorehabilitation non-trivial to ensure patient safety, with multiple dynamical features needing to be determined. Global entrainment mechanisms, for example, must be distinguished from the motor attractor so that they are not modified by the activity of the implants, as should contiguous attractors that may be activated. What this seems to point to collectively is that the why question of global nervous function cannot be neglected when designing devices suited to brain implementation. Indeed, the overcoming of noise is only one consideration that is introduced by philosophical considerations on living, particularly human, organisms. Autonomy implies that goal seeking must be integrated in light of the holistic nature of the organism. That is, the nervous system must be conceived as an entity. To be otherwise, would eventually result in its complete disintegration, as evidenced in non-living systems. In fact there is increasing evidence that the dynamics of the brain are so constructed to enable this unitary and integral character to be the organism's pre-eminent form, to which all neural processes are subordinate. The construction of stable elements like attractor motifs subserve this objective and is akin to the construction of building blocks that can then be assembled to create global platforms that are capable of eliciting localized activity in the context of the whole, or serve as the basis for personal identity. Fortunately, there is a growing recognition on the part of the neuroscientific community that is reflected in current trends to identify global models for such features as consciousness, sleep, personal identity, and control over motor behaviors $[18,19]$. In a phrase, a supraphysical conception is not just a bad penny that keeps showing its image indiscretely, but a determinative feature of nature that anchors its appearance. Hopefully, neuro-engineers will soon adopt this view too.

\section{References}

1. Córdova-Palomera A, Kaufmann T, Persson K, Alnæs D, Doan NT, et al. (2017) Disrupted global metastability and static and dynamic brain connectivity across individuals in the Alzheimer's disease continuum. Sci Rep 7: 40268. [Crossref]

2. Hellyer PJ, Shanahan M, Scott G, Wise RJ, Sharp DJ, et al. (2014) The control of global brain dynamics: opposing actions of frontoparietal control and default mode networks on attention. J Neurosci 34: 451-461. [Crossref]

3. Wu J, Raver C, Piao C, Keller A, Faden AI (2013) Cell cycle activation contributes to increased neuronal activity in the posterior thalamic nucleus and associated chronic hyperesthesia after rat spinal cord contusion. Neurotherapeutics 10: 520-538. [Crossref]

4. Chen Y, Li G, Zhu Y, Zhao J, Cai H (2014) Design of a 6-DOF upper limb rehabilitation exoskeleton with parallel actuated joints. Biomed Mater Eng 24: 2527-2535. [Crossref]

5. Grafstein B (1980) Cellular mechanisms for recovery from nervous system injury. Surg Neurol 13: 363-365. [Crossref]

6. Kahlaoui K, Wilson M, Ansaldo AI, Ska B, Joanette Y (2011) Neurobiological and neuroethical perspectives on the contribution of functional neuroimaging to the study of aging in the brain. In: Illes J, Sahakian B, editors. Oxford Handbook of Neuroethics. Oxford: Oxford Press.

7. Feigin VL, Forouzanfar MH, Krishnamurthi R, Mensah GA, Connor M, et al. (2014) Global and regional burden of stroke during 1990-2010: findings from the Global Burden of Disease Study 2010. Lancet 383: 245-254. [Crossref]

8. Cong P (2016) Neural interfaces for implantable medical devices: Circuit design considerations for sensing, stimulation, and safety. IEEE Solid-States Circuits Magazine Fall: 48-56.

9. Jensen W, Andersen OK, Akay M. (2014) Replace, Repair, Restore, Relieve - Bridging Clinical and Engineering Solutions. Aalborg, Denmark: Springer International Publishing.

10. Cong P, Ibid. Jackson A, Hall TM (2010) Decoding local field potential for neural interfaces. IEEE Trans Neural Systems Rehabilitation Engineering: 1-10.

11. Schoner G (2009) Development as change of system dynamics: Stability, instability, and emergence. In: Spencer JP, Thomas MSC, McClelland JL, editors. Toward a Unified Theory of Development: Connectionism and Dynamic Systems Theory Reconsidered. Oxford: Oxford University Press.

12. Reimann H, Lins J, Schoner G (2015) The dynamics of neural activation variables. $J$ Behavioral Robotics 6: 57-70.

13. McClelland JL, Vallabha G (2009) Connectionist models development: mechanical, dynamical models with emergent dynamical properties. In: Toward a Unified Theory of Development: Connectionism and Dynamic Systems Theory Re-considered, J.P. Spencer, M.S.C. Thomas, and J.L. McClelland Eds. Oxford: Oxford University Press.

14. Reimann H, Lins J, Schoner, Ibid. 
15. Kato S, Kaplan HS, Schrödel T, Skora S, Lindsay TH, et al. (2015) Global brain dynamics embed the motor command sequence of Caenorhabditis elegans. Cell 163 : 656-669. [Crossref]

16. Smith L (2009) Stability and flexibility in development. In: Spencer JP, Thomas MSC, McClelland JL, editors. Toward a Unified Theory of Development: Connectionism and Dynamic Systems Theory Re-considered. Oxford: Oxford University Press.
17. Tononi G, Sporns O, Edelman GM (1994) A measure for brain complexity: relating functional segregation and integration in the nervous system. Proc Natl Acad Sci U S A 91: 5033-5037. [Crossref]

18. Benson AR, Gleich DF, Leskovec J (2016) Higher-order organization of complex networks. Science 353: 163-166. [Crossref]

Copyright: $\mathbb{0} 2017$ Larrivee D. This is an open-access article distributed under the terms of the Creative Commons Attribution License, which permits unrestricted use, distribution, and reproduction in any medium, provided the original author and source are credited. 